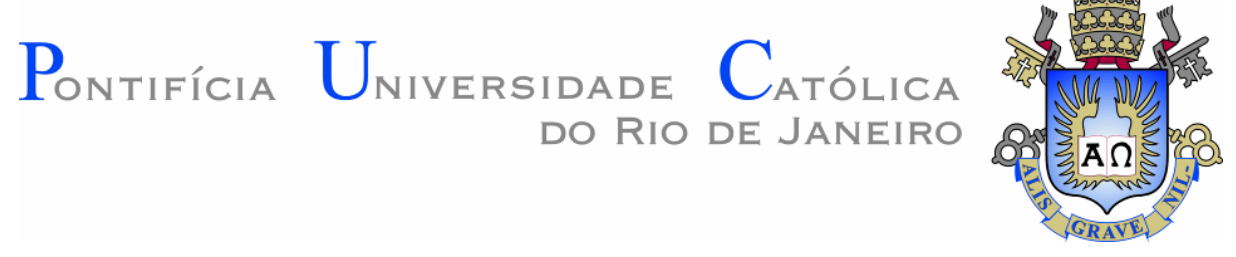

Leydi del Rocío Silva Calpa

\title{
Estudo Preliminar da Sinterização de Membranas Macroporosas de Óxido de Magnésio
}

Dissertação de Mestrado

Dissertação apresentada como requisito parcial para obtenção do grau de Mestre pelo Programa de PósGraduação em Engenharia de Materiais e de Processos Químicos e Metalúrgicos da PUC-Rio.

Orientador: Prof. Roberto Ribeiro de Avillez Co-orientador: Prof. Sidnei Paciornik 


\section{Leydi del Rocío Silva Calpa}

\section{Estudo Preliminar da Sinterização de Membranas Macroporosas de Óxido de Magnésio}

Dissertação apresentada como requisito parcial para obtenção do grau de Mestre pelo Programa de Pósgraduação em Engenharia de Materiais e de Processos Químicos e Metalúrgicos do Departamento de Engenharia de Materiais do Centro Técnico Científico da PUC-Rio. Aprovada pela Comissão Examinadora abaixo assinada.

Prof $^{\circ}$. Roberto Ribeiro de Avillez Orientador e Presidente

Departamento de Engenharia de Materiais - PUC-Rio

Prof $^{\circ}$. Sidnei Paciornik Co-Orientador Departamento de Engenharia de Materiais - PUC-Rio

Prof ${ }^{a}$. Paula Mendes Jardim Universidade Federal do Rio de Janeiro-UFRJ

Prof ${ }^{\circ}$. Bojan Marinkovic Departamento de Engenharia de Materiais - PUC-Rio

Prof. José Eugênio Leal

Coordenador Setorial de Pós-Graduação do Centro Técnico Científico da PUC-Rio 
Todos os direitos reservados. É proibida a reprodução total ou parcial do trabalho sem autorização da universidade, da autora e do orientador.

\section{Leydi del Rocío Silva Calpa}

Formada em Química na Universidade de Nariño (Colômbia), onde foi integrante do grupo de pesquisa em Materiais Funcionais e Catálise. Atualmente finalizando o curso de mestrado em Engenharia dos Materiais, Processos Químicos e Metalúrgicos na Pontifícia Universidade Católica de Rio de Janeiro PUC-Rio, onde desenvolve pesquisa sobre a obtenção de materiais cerâmicos macroporosos de $\mathrm{MgO}$.

Ficha Catalográfica

Calpa, Leydi del Rocío Silva

Estudo preliminar da sinterização de membranas macroporosas de óxido de magnésio / Leydi del Rocío Silva Calpa ; orientador: Roberto Ribeiro de Avillez ; coorientador: Sidnei Paciornik. - 2011.

109 f. : il. (color.) ; $30 \mathrm{~cm}$

Dissertação (mestrado)-Pontifícia Universidade Católica do Rio de Janeiro, Departamento de Engenharia de Materiais, 2011.

Inclui bibliografia

1. Engenharia de materiais - Teses. 2. Materiais nanoestruturados. 3. Óxido de magnésio. 4. Materiais macroporosos. 5. Sinterização. I. Avillez, Roberto Ribeiro de. II. Paciornik, Sidnei. III. Pontifícia Universidade Católica do Rio de Janeiro. Departamento de Engenharia de Materiais. IV. Título. 
Com amor dedico este trabalho a os meus pais Angel e Isabel, a minhas irmãs Alicia, Floralba, Magaly e Greis, a os meus sobrinhos Gabriel, Carolina, Daniela, Viviana, Camila, Alejandra, Santiago y Karlita quem sempre estão perto de mim mesmo na distância, dando-me seu apoio e amor, me ajudando de todas as formas possíveis e, sobretudo me fazendo sentir motivação cada dia. À Greis, que mais que uma irmã, é uma grande amiga; ela tem me acompanhado, apoiado, escutado e aconselhado carinhosa, paciente e incondicionalmente. Com grande amor para essa família maravilhosa que Deus me deu. 


\section{Agradecimentos}

Aos meus orientadores Roberto de Avillez e Sidnei Paciornik pela orientação, dedicação e apoio no desenvolvimento do presente trabalho.

Ao CNPq e à PUC-Rio, pelos auxílios concedidos, sem os quais este trabalho não poderia ter sido realizado.

Aos professores que fizeram parte da comissão examinadora.

A todas as pessoas da PUC-Rio cuja intervenção foi de grande importância para o desenvolvimento do presente trabalho: à Maria Isabel e ao Johnny Huertas do departamento de Química, ao Sergio Fontoura e ao Víctor Aréstegui do departamento da Engenharia Civil, ao Ronaldo Pedro do laboratório de DRX DEMa; ao André, o Maurício e o Marcos Enrique do laboratório de microscopia eletrônica de varredura - DEMa, ao Alexandre Bueno pelas explicações fornecidas e pelo carácter serviçal e atento em todo momento.

À Greis, ao Josemi M. e Andrés Gaona por estar presentes nos momentos más difíceis, pelos conselhos e momentos compartidos, e por estar sempre atentos á evolução do meu trabalho. À Andrea, Lorena, Sandra, David, Dasha, Gerardo, Cesar ao Andrew ao Jorge, ao Fredy, a todos meus colegas da PUC-Rio, a todos os que me ajudaram incondicionalmente.

Agradeço à minha família, cada carta, cada mensagem de motivação, cada palavra, cada desejo permanecem sempre no meu coração me alentando. 


\section{Resumo}

Calpa, Leydi del Rocío Silva; de Avillez, Roberto Ribeiro; Paciornik, Sidnei. Estudo Preliminar da Sinterização de Membranas Macroporosas de Óxido de Magnésio. Rio de Janeiro, 2011. 109p. Dissertação de Mestrado - Departamento de Engenharia de Materiais, Pontifícia Universidade Católica do Rio de Janeiro.

O presente trabalho apresenta um estudo preliminar sobre a síntese de membranas macroporosas de óxido de magnésio, com uma faixa de porosidade estreita e homogênea, mediante uma técnica reprodutível e visando sua aplicação futura na separação de substâncias gasosas impossíveis de serem separadas por métodos convencionais. As membranas foram criadas a partir da sinterização das respetivas nanopartículas obtidas pelo método sol-gel/nano matriz, usando nitrato de magnésio como precursor principal e álcool polivinílico, PVA, como matriz das nanoestruturas. Foram avaliados diferentes parâmetros de pressão e temperatura de sinterização. As amostras obtidas foram analisadas por MEV, DRX, BET e Porosimetria por Intrusão de Mercúrio (PIM). Os resultados mostram que o material obtido apresenta tamanho de partículas uniforme, confirmando assim a sinterização. O tamanho de cristalito médio foi de $\sim 160 \mathrm{~nm}$, sendo que o tamanho médio de grãos foi de 450nm; os materiais que apresentaram melhores propriedades foram aqueles sinterizados a 1000 e $1100^{\circ} \mathrm{C}$ com pressão de $173 \mathrm{MPa}$, e a $1000^{\circ} \mathrm{C}$ com $260 \mathrm{MPa}$. A macroporosidade obtida se encontra numa faixa muito perto do limite com a mesoporosidade, sendo que quase $97 \%$ dos macroporos estão entre 50 e 150nm de raio. Os resultados indicam que a técnica foi reprodutível e a faixa de poros é razoavelmente estreita, o que permitirá aplicar a membrana na separação de substâncias específicas.

\section{Palavras-chave}

Materiais nanoestruturados; óxido de magnésio; materiais macroporosos; sinterização. 


\section{Abstract}

Calpa, Leydi del Rocío Silva; de Avillez, Roberto Ribeiro (Advisor); Paciornik, Sidnei. Preliminary study of magnesium oxide macroporous membrane sintering. Rio de Janeiro, 2011. 109p. MSc dissertation Departamento de Engenharia de Materiais, Pontifícia Universidade Católica de Rio de Janeiro.

This work present a preliminary study on the synthesis of macroporous membranes of magnesium oxide with a homogeneous and a narrow range of porosity obtained by a reproducible technique, which aims their future application in the separation of gaseous substances impossible to separate by conventional techniques. The membranes were obtained by the sintering of the respective nanoparticles which were obtained by the sol-gel/nanotemplate technique, using a magnesium nitrate as the main precursor and polyvinyl alcohol, PVA, as a template of the nanostructures. Pressure and Temperature were the sintering parameters evaluated in the present research. The samples obtained were analyzed by SEM, XRD, BET, and Intrusion Mercury Porosimetry (IMP). The results show uniformity in the particles size of the material, confirming the sintering process. The average crystallite size was $160 \mathrm{~nm}$, and the average grain size was $450 \mathrm{~nm}$, the samples showing better properties were those sintered at 1000 and $1100^{\circ} \mathrm{C}$ at a pressure of $173 \mathrm{MPa}$, and $1000^{\circ} \mathrm{C}$ at $260 \mathrm{MPa}$. The range macroporosity obtained limit with the mesoporosity, nearly $97 \%$ of the macropores is between 50 and $150 \mathrm{~nm}$ radius. The results indicate that the technique is reproducible and the pores range is reasonably small, this will allow to apply the membrane in the separation of certain substances.

\section{Key-words}

Nanostructured materials, magnesium oxide, macroporous materials, sintering. 


\section{Sumário}

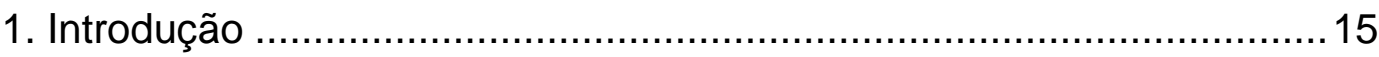

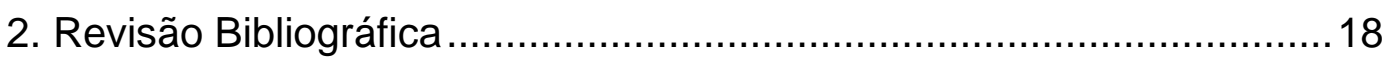

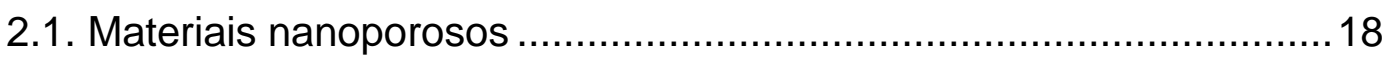

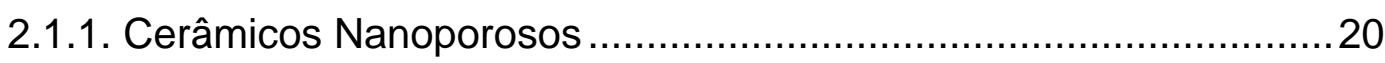

2.2. Nanomembranas Cerâmicas ...........................................................21

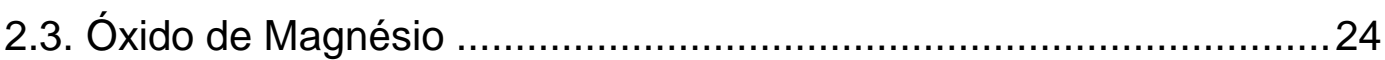

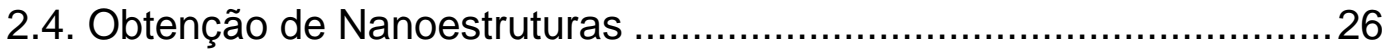

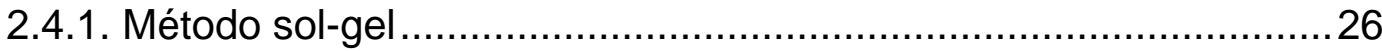

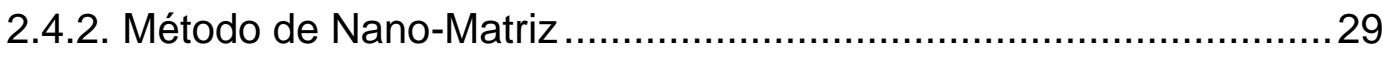

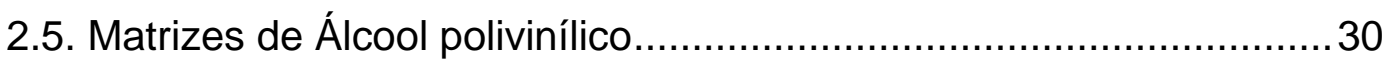

2.6. Sinterização e seus parâmetros na obtenção de nanoestruturas......32

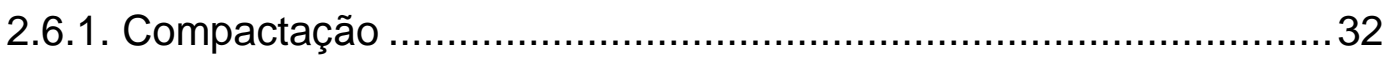

2.6.1.1. Primeiro passo: Preenchimento do molde ...................................34

2.6.1.2. Segundo passo: compactação ou prensagem ............................34

2.6.1.3. Terceiro passo: desmoldagem ................................................. 35

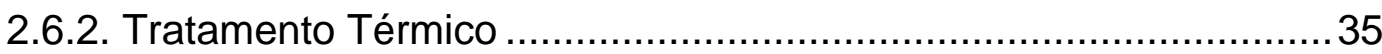

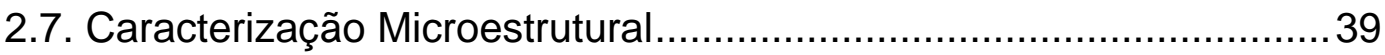

2.8. Determinação de Área Superficial pela Técnica BET .........................40

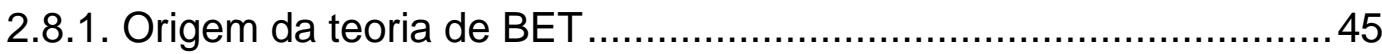

2.9. Porosimetria por Injeção de Mercúrio PIM .....................................54

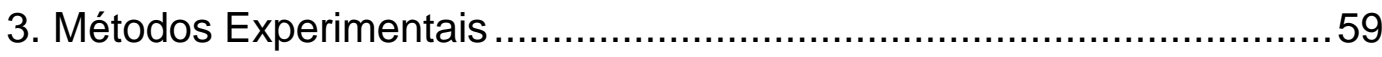

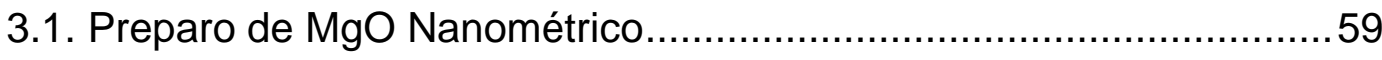




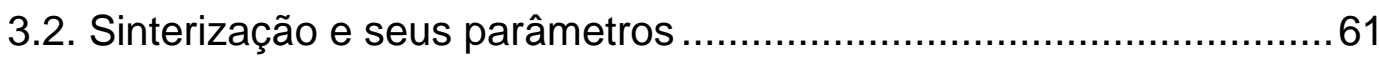

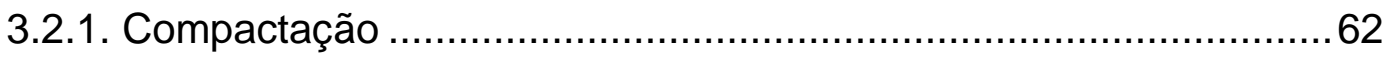

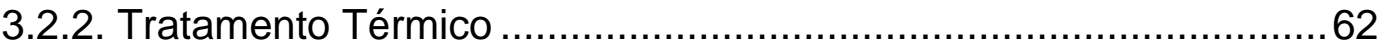

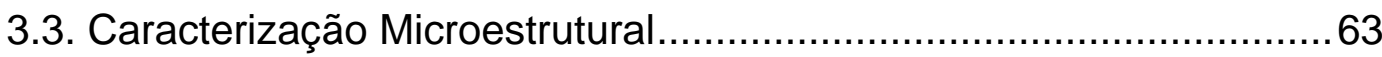

3.3.1. Microscopia Eletrônica de Varredura MEV .....................................64

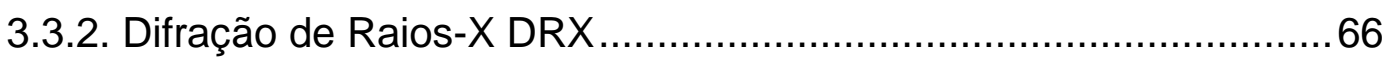

3.3.3. Determinação da área superficial pela técnica BET .......................67

3.3.4. Porosimetria por Injeção de Mercúrio..............................................67

4. Resultados e Análise dos Resultados ..............................................69

4.1. Microscopia Eletrônica de Varredura MEV .......................................69

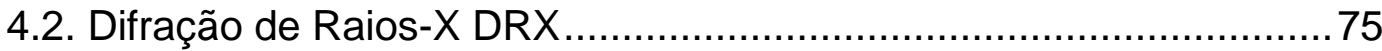

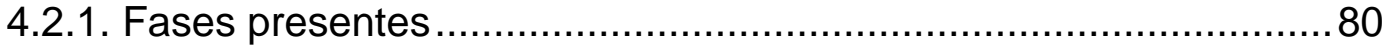

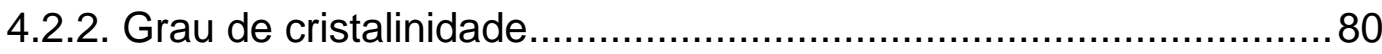

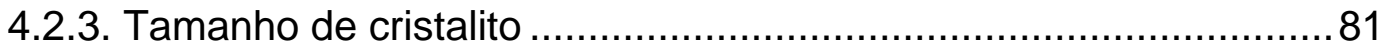

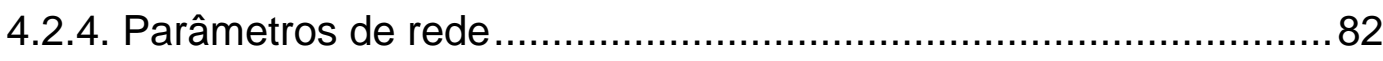

4.3. Determinação da área superficial pela técnica BET ......................... 82

4.4. Porosimetria por Injeção de Mercúrio ................................................94

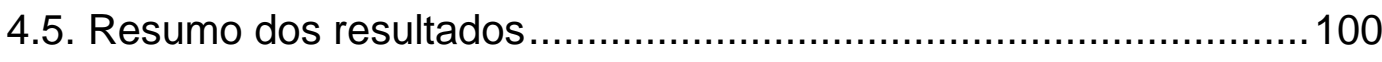

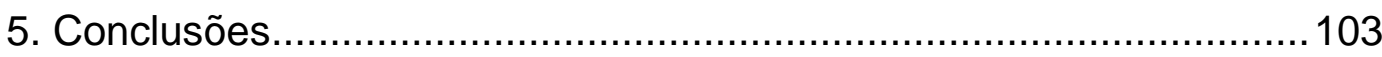

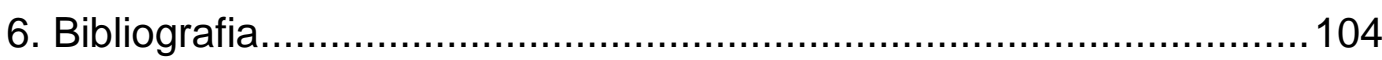




\section{Lista de Figuras}

Figura 2.1 - Representação esquemática de alguns tipos de poros. a) fechados, b) abertos, c) abertos penetrantes, d) tipo garrafa, e) tipo funil

Figura 2.2 - Esquema do preenchimento, prensagem e desmoldagem do material verde no processo de prensagem uniaxial de partículas de pó seco sem adição de adjuvantes

Figura 2.3 - Coalescência de dois grãos de tamanhos ligeiramente diferentes a) partículas em contato antes da coalescência b) crescimento do pescoço gerado entre as partículas, crescimento do grão (KANG, 2007).

Figura 2.4 Geometria dos poros segundo seu número de coordenação $\mathrm{N}$ ou número de grãos que o circundam. a) $\mathrm{N}<6$ lados convexos, tendência ao encolhimento do poro b) $\mathrm{N}=6$ lados retos, estrutura metaestável c) N>6 lados côncavos, tendência ao crescimento do poro.

Figura 2.5 - Representação esquemática dos seis tipos de isotermas de adsorção. Setas azuis indicam laços de histerese, setas vermelhas, começo de multicamada (KANG, 2007)

Figura 2.6 Tipos de laços ou loop de histerese presentes tipicamente nas isotermas de adsorção tipo IV e V.

Figura 2.7 Esquema porosidade interna de um material que está sendo preenchido por mercúrio. Regiões 1-4 representam preenchimento com pressões incrementais sucessivas. A região 5 corresponde a um poro fechado não preenchido na analise. .56

Figura 3.1 esquema da formação do sistema sol-gel e da matriz polimérica. Adição dos dois sistemas com os tratamentos térmicos adequados e a consequente formação das nanopartículas de $\mathrm{MgO}$ 
Figura 3.2 Imagem esquerda) sol-gel de composição PVA + $\mathrm{Mg}(\mathrm{NO} 3) 2$. Direita) forno usado no tratamento térmico do material a $200^{\circ} \mathrm{C}$ e $600^{\circ} \mathrm{C}$.

Figura 3.3 a) matriz de aço para a formação de pastilhas do material em pó. b) prensa mecânica empregada para a compactação do pó depositado dentro da matriz de aço

Figura 3.4 Disposição do cadinho de cerâmica dentro do tubo do forno, no processo de tratamento térmico das amostras. Imagem esquerda: inserção lenta do cadinho

Figura 4.1 Imagem do $\mathrm{MgO}$ após tratamento térmico a $600^{\circ} \mathrm{C}$ (eliminação do PVA)

Figura 4.2 Imagem do $\mathrm{MgO}$ após tratamento térmico sucessivo a $200^{\circ} \mathrm{C} / 1 \mathrm{~h}, 600^{\circ} \mathrm{C} / 1 \mathrm{~h}$ e $1000^{\circ} \mathrm{C} / 1 \mathrm{~h}$. esquerda) aumento $2000 \mathrm{X}$, direita) aumento $10000 \mathrm{X}$

Figura 4.3 Vista do corte transversal manual da amostra (P2000 S1000). Microscópio operado a 20kV. Superior) ampliação 3000X, inferior) ampliação 15000X.

Figura 4.4 Vista do corte transversal da amostra P2000 S1000, feito com faca de diamante prévio embutimento com metil metacrilato. Microscópio operado em alto vácuo, sinal SEI, 20kV, e spot 42.

Figura 4.5 Vista do corte transversal da amostra P2000 S1000. Corte feito prévio embutimento com Epothin Epoxy Resin $\AA$, lixamento sequencial com lixas \# 1000 e 1200, polimento automático com panos com pasta de diamante de $6 \mu \mathrm{m}, 3 \mu \mathrm{m}$ e $1 \mu \mathrm{m}$. Microscópio operado em alto vácuo, sinal SEI, 20kV, e spot 42.

Figura 4.6 Vista do corte transversal da amostra P2000 S1000. Corte transversal obtido por lixamento sequencial e polimento automático com panos a base de diamante de $6 \mu \mathrm{m}$, $3 \mu \mathrm{m}$ e $1 \mu \mathrm{m}$ e pasta de diamante. Microscópio operado em alto vácuo, sinal SEI, $20 \mathrm{kV}$, e spot 42 . 
Figura 4.7 Difratograma da amostra de MgO (P2000 S900). Radiação usada: CrKa , faixa $40-120^{\circ} 2 \theta$, tempo de aquisição de 3 segundos/passo.

Figura 4.8 Difratograma da amostra de MgO (P2000 S1000). Radiação usada: CuKa , faixa $20-90^{\circ} 2 \theta$, tempo de aquisição de 3 segundos/passo.

Figura 4.9 Difratograma da amostra de MgO (P2000 S1100). Radiação usada: CuKa , faixa $20-90^{\circ} 2 \theta$, tempo de aquisição de 3 segundos/passo.

Figura 4.10 Difratograma da amostra de MgO (P2000 S1100). Radiação usada: CuKa , faixa $20-90^{\circ} 2 \theta$, tempo de aquisição de 3 segundos/passo.

Figura 4.11 Difratograma da amostra de MgO (P3000 S900). Radiação usada: CuKa , faixa $40-120^{\circ} 2 \theta$, tempo de aquisição de 3 segundos/passo.

Figura 4.12 Difratograma da amostra de MgO (P3000 S1000). Radiação usada: CuKa , faixa $40-120^{\circ} 2 \theta$, tempo de aquisição de 3 segundos/passo.

Figura 4.13 Difratograma da amostra de MgO (P3000 S1100). Radiação usada: CuKa , faixa $20-90^{\circ} 2 \theta$, tempo de aquisição de 3 segundos/passo. .79

Figura 4.14 Difratograma da amostra de MgO (P3000 S1100). Radiação usada: CuKa , faixa $20-90^{\circ} 2 \theta$, tempo de aquisição de 3 segundos/passo.

Figura 4.15 Parâmetro de rede "a" para cada sistema, como função da sua pressão de compressão e temperatura de sinterização.

Figura 4.16 Isoterma de Adsorção/desorção de Nitrogênio sobre a amostra de MgO (P2000 S900). 83

Figura 4.17 Isoterma de Adsorção/desorção de Nitrogênio sobre a amostra de MgO (P2000 S1000)

Figura 4.18 Isoterma de Adsorção/desorção de Nitrogênio sobre a amostra de MgO (P2000 S1100). 
Figura 4.19 Isoterma de Adsorção/desorção de Nitrogênio sobre a amostra de MgO (P2000 S1200).

Figura 4.20 Isoterma de Adsorção/desorção de Nitrogênio sobre a amostra de MgO (P3000 S900)..

Figura 4.21 Isoterma de Adsorção/desorção de Nitrogênio sobre a amostra de MgO (P3000 S1000).

Figura 4.22 Isoterma de Adsorção/desorção de Nitrogênio sobre a amostra de MgO (P3000 S1100).

Figura 4.23 Isoterma de Adsorção/desorção de Nitrogênio sobre a amostra de MgO (P3000 S1200).

Figura 4.24 Área Superficial Específica BET das amostras estudadas, em função da pressão de compressão e da temperatura de sinterização.

Figura 4.25 Distribuição de Tamanho de Poros na amostra (P2000 S900) determinada a partir da isoterma de adsorção de nitrogênio, pela técnica BJH.

Figura 4.26 Distribuição de Tamanho de Poros na amostra (P2000 S1000) determinada a partir da isoterma de adsorção de nitrogênio, pela técnica BJH

Figura 4.27 Distribuição de Tamanho de Poros na amostra (P2000 S1100) determinada a partir da isoterma de adsorção de nitrogênio, pela técnica BJH 90

Figura 4.28 Distribuição de Tamanho de Poros na amostra (P2000 S1200) determinada a partir da isoterma de adsorção de nitrogênio, pela técnica BJH 90

Figura 4.29 Distribuição de Tamanho de Poros na amostra (P3000 S900) determinada a partir da isoterma de adsorção de nitrogênio, pela técnica BJH

Figura 4.30 Distribuição de Tamanho de Poros na amostra (P3000 S1000) determinada a partir da isoterma de adsorção de nitrogênio, pela técnica BJH

Figura 4.31 Distribuição de Tamanho de Poros na amostra (P3000 S1100) determinada a partir da isoterma de adsorção de nitrogênio, pela técnica BJH 
Figura 4.32 Distribuição de Tamanho de Poros na amostra (P3000 S1200) determinada a partir da isoterma de adsorção de nitrogênio,

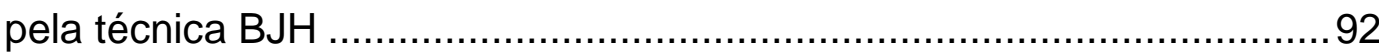

Figura 4.33 Comparação das distribuições de tamanho de poros nos sistemas trabalhados, determinadas por analise BJH na técnica de adsorção de $\mathrm{N}_{2}$

Figura 4.34 Distribuição de Tamanho de Poros na amostra (P2000 S900) determinada a partir da técnica PIM.

Figura 4.35 Distribuição de Tamanho de Poros na amostra (P2000 S1000) determinada a partir da técnica PIM.

Figura 4.36 Distribuição de Tamanho de Poros na amostra (P2000 S1100) determinada a partir da técnica PIM. 96

Figura 4.37 Distribuição de Tamanho de Poros na amostra (P2000 S1200) determinada a partir da técnica PIM. .96

Figura 4.38 Distribuição de Tamanho de Poros na amostra (P3000 S1000) determinada a partir da técnica PIM.

Figura 4.39 Histograma PIM dos raios dos poros na amostra P2000 5900

Figura 4.40 Histograma PIM dos raios dos poros na amostra P2000 S1000

Figura 4.41 Histograma PIM dos raios dos poros na amostra P2000 S1100. 99

Figura 4.42 Histograma PIM dos raios dos poros na amostra P2000 S1200. 99

Figura 4.43 Histograma PIM dos raios dos poros na amostra P3000 S1000. 